\title{
Investigation and management of long-standing chronic constipation in childhood
}

\author{
G. S. CLAYDEN and J. O. N. LAWSON \\ From St. Thomas's Hospital and Westminster Children's Hospital, London
}

\begin{abstract}
Clayden, G. S., and Lawson, J. O. N. (1976). Archives of Disease in Childhood, 51, 918. Investigation and management of long-standing chronic constipation in childhood. The anorectal physiology of 106 children with long-standing chronic constipation, who had failed to respond to a trial of medical treatment, was assessed. $10(9 \%)$ were shown to have ultrashort-segment Hirschsprung's disease, later confirmed on histology. The remainder showed evidence of hypertrophy of the internal sphincter on anorectal manometry and had a vigorous anal dilatation (to accept 4 fingers) under general anaesthesia. After this, $38 \%$ were able to be weaned off all medication and most of the remainder improved. Further anal dilatation and internal sphincterotomy allowed a further 10 children to stop laxatives, bringing the total to $48 \%$.
\end{abstract}

Chronic constipation, especially when associated with faecal impaction and soiling, is a common and distressing condition in childhood, affecting not only the child but also his family. The general concern and distress is imparted to the doctor who is usually attempting management without a precise diagnosis. There is a basic lack of agreement even in nomenclature, and advice from paediatrician, psychiatrist, or surgeons varies. Some advise an active approach with repeated enemata and laxatives, others even advise ignoring the problem. Though spontaneous resolution occurs, the more common evolution is that soiling gradually undermines the child's and the family's well being, especially when it becomes obvious to his associates at school. Should the symptoms be caused by Hirschsprung's disease there is a real risk of the serious consequences of enterocolitis.

We have concentrated on the child who presents with chronic constipation (difficulty or delay in defecation) with or without soiling (inadvertent passage of loose faeces into clothing) who have failed to respond to medical treatment, including in most cases psychiatric attention. To achieve a better understanding of the pathophysiology, anorectal manometry was used, which is a safe and relatively noninvasive technique.

The system, developed between 1965 and 1967,

Received 8 March 1976. was originally designed to investigate the squeeze pressure changes in the anal canal and rectum of normal children and those suffering from Hirschsprung's disease (Lawson and Nixon, 1967; Lawson, 1974). In the original series (Lawson and Nixon, 1967; Lawson, 1974) children thought likely to be suffering from Hirschsprung's disease were investigated. The changes in 24 normal and 63 cases of Hirschsprung's disease were reported. Of the cases presenting at this time with chronic constipation, 1 child in 5 was found to be suffering from Hirschsprung's disease. This suggested that Hirschsprung's disease, particularly of the ultrashort-segment variety (Lawson, 1970), might be a potent cause of long-standing chronic constipation. It became clear that after excluding those cases with organic obstruction or constipation resulting from a previously unsuspected spinal anomaly most cases of chronic constipation had changes suggesting a functional obstructive element. We therefore studied a group of patients who had not been selected as likely cases of Hirschsprung's disease.

\section{Patients and methods}

106 cases of chronic constipation, the majority with histories dating back to birth or the first year of life, were studied. These were children who failed to respond to an initial trial of medical treatment.

A full history was taken to include details of the complaints, age of onset, associated urinary symptoms, family 
TABLE I

\section{Presenting complaints of the 106 children with chronic constipation}

\begin{tabular}{lr} 
Constipation without soiling & 41 \\
Constipation with soiling & 61 \\
Soiling alone & 4 \\
\hline
\end{tabular}

history, and obstetric, neonatal, and past history. Fig. 1 shows the age of presentation and Table $I$ the frequency of the presenting complaints. Each child was examined in detail to assess the degree of faecal retention, the degree of abdominal distension, organic obstruction (anal stenosis and anal anomaly), unsuspected spinal lesions (sacral agenesis, scimitar sacrum, and spinal dysrhaphism), and any evidence of urinary tract anomalies.

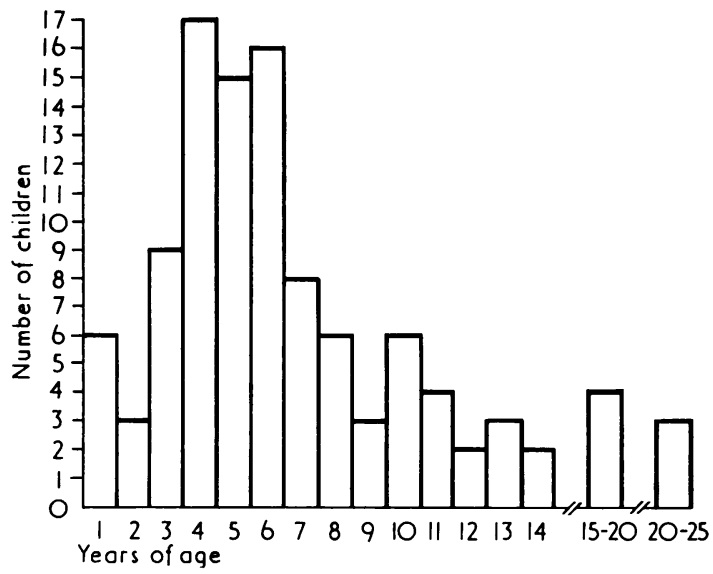

FIG. 1.-Age of presentation of children with chronic constipation.

Investigations. Each child was investigated by one or more of the following methods.

Barium enema on unprepared bowel. Initially most cases presenting with long-term constipation were subjected to this investigation. However, even in the most experienced hands the findings, apart from the enlarged rectum, were extremely difficult to interpret. In general the diagnosis of ultrashort-segment Hirschsprung's disease by barium enema was very unreliable, its main use being an assessment of rectal size.

Anorectal physiology. All the cases in this series were investigated by the following method, though some cases with a clear history of anal fissure or more recent onset of constipation were given a trial of medical management first. The method, based on a balloon system first described by Denny-Brown and Robertson (1935) and later by Gaston (1948), Schuster et al. (1965), and Schnaufer et al. (1967), was developed to give a simultaneous record of a squeeze pressure profile of the anal canal and rectum.

Probes, made of turned brass and stainless steel tubes, consist of four rubber covered chambers $(0.5 \times 0.4 \mathrm{~cm}$ and $1.0 \times 0.5 \mathrm{~cm})$. These record at $0.5 \mathrm{~cm}$ or $1.0 \mathrm{~cm}$ intervals through the anal canal and into the lower rectum. The smaller probe, designed for the newborn and infant, the larger for older children, accords with Schapiro's (1948) account of anal and rectal size in childhood. A latex balloon set on a rigid spindle is fixed with a flexible tube to one end of the probe and lies in the rectum $5 \mathrm{~cm}$ or $7 \mathrm{~cm}$ from the anal verge. It is used to record rectal pressure and also to distend the rectum. The chambers and rectal balloon are connected to transducers by stainless steel and nylon tubes and via amplifiers to the UV recorder (SE oscillograph 3006). In addition an electromyogram from the perineum and $a$ pneumograph are recorded. The impulses are also recorded on a data magnetic tape for subsequent wave analysis on a digital computer (Varian 620/L-100).

The system, originally air-filled, was converted to a water-filled one when the apparatus was redesigned in 1970, though the basic principles remain unaltered. The pressures at rest and the changes in pressure in the anal canal with rectal distension, with perianal skin stimulation, and with movement of the probe in the anal canal are recorded. In addition, the subjective sensation with rectal distension is noted where possible.

Physiological findings. In the cases of chronic constipation reported here, of the 10 traces which showed the characteristics of Hirschsprung's disease, diagnosis was confirmed in 8 by mucosal biopsy. In the other 2, though the mucosal biopsy contained ganglion cells, a subsequent specimen obtained at extended sphincterotomy showed no ganglion cells in the muscle plexus for 4 to $5 \mathrm{~cm}$ from the anal verge (a finding confirmed in a personal communication from $\mathrm{H}$. Beardmore, Montreal). Ten other traces were considered suspicious of ultrashort-segment Hirschsprung's disease, but in these biopsy failed to show absence of ganglion cells or abnormal nerve bundles.

Physiological findings in the other 79 cases were abnormal, but were not characteristic of Hirschsprung's disease. The trace taken from the internal sphincter zone showed prominent amplitude rhythmical activity, superimposed on the slow fluctuations, as seen in Hirschsprung's disease, but a fall in pressure accompanied spontaneous, and often hypertrophic, rectal waves. Similarly, there was a fall in pressure in the internal sphincter zone, corresponding to a stimulated rectal wave, with rectal distension. This response was delayed until larger volumes had been introduced into the rectal balloon. Also the critical volume (with the sensation of urgent desire to defecate) was markedly increased, so that complete inhibition was not reached until 200$300 \mathrm{ml}$ were introduced into the distensible rectal balloon (normal, $150 \mathrm{ml}$ after infancy). The delay in inhibitory response and failure to reach the critical volumes appeared to be a result of the megarectum. 
The hypertrophic rectal waves suggest rectal hypertrophy rather than 'inertia'.

In most of these cases, though the inhibition appeared to be as profound as the normal in upper chambers, they had a less complete fall in the first $\mathrm{cm}$. There was also a more abrupt and exaggerated return of pressure after the introduction of the initial volumes of air into the rectal balloon.

Four of the 79 cases on anal dilatation were shown to have a minor degree of anal stenosis, with a string stricture at the mucocutaneous junction. This string stricture, approximately $1 \mathrm{~cm}$ from the anal verge, accepted only 2 fingers of the operator's hand during anal dilatation. Those in this group who did not show a Hirschsprung-like trace but whose symptoms went back to the neonatal period or infancy could be termed 'congenital constipation' or 'anal achalasia', depending on the age of onset of symptoms ('anal achalasia' is used to indicate the obstructive nature of the internal sphincter).

\section{Results}

Using the investigation schedule described, 106 patients who presented with chronic constipation fell into the following groups: 10 newly discovered ultrashort-segment Hirschsprung's disease; 85 'anal achalasia' (congenital constipation); 4 minor anal strictures; 4 idiopathic myenteric plexus dysfunction; 1 scimitar sacrum; 1 sacral agenesis; 1 coeliac disease.

Clinical features in anal achalasia and ultrashort-segment Hirschsprung's disease. Age of onset of symptoms of constipation is shown in Fig. 2. The sex incidence of anal achalasia was 63 males to 24 females $(2 \cdot 6: 1)$, and of ultrashortsegment Hirschsprung's disease 6 males to 4 females $(1 \cdot 5: 1)$. Symptoms present in patients with

\section{TABLE II}

\section{Symptoms}

\begin{tabular}{|c|c|c|}
\hline & $\begin{array}{c}\text { Anal } \\
\text { achalasia } \\
(\mathrm{n}=\mathbf{8 5})\end{array}$ & $\begin{array}{l}\text { Ultrashort- } \\
\text { segment } \\
\text { Hirsch- } \\
\text { sprung's } \\
(n=10)\end{array}$ \\
\hline $\begin{array}{l}\text { Onset from infancy } \\
\text { Stool frequency: } \\
3-\text { to 10-day interval } \\
10+\text { days interval } \\
\text { Difficulty with defecation } \\
\text { Soiling } \\
\text { Abdominal distension } \\
\text { Urinary symptoms } \\
\text { Skin problems } \\
\text { (eczema/icthyosis) } \\
\text { Neonatal obstruction } \\
\text { Strong family history of } \\
\text { constipation } \\
\text { Overt psychological } \\
\text { problems }\end{array}$ & $\begin{array}{r}49 \\
39 \\
15 \\
45 \\
51 \\
27 \\
16 \\
5 \\
3 \\
25 \\
20\end{array}$ & $\begin{array}{l}9 \\
5 \\
4 \\
5 \\
4 \\
8 \\
2 \\
1 \\
4 \\
0 \\
2\end{array}$ \\
\hline
\end{tabular}

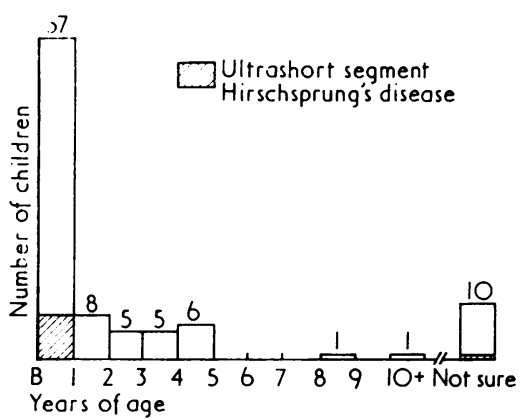

FIG. 2.-Age of onset of symptoms of constipation.

achalasia and Hirschsprung's disease are given in Table II.

The family history was strongly positive in 25 of the non-Hirschsprung cases and it is of interest that the mother and the maternal grandparents were the most frequently affected.

Management. Over the past 5 years it had been our policy to adhere to a phased approach to treatment: (i) medical management, (ii) trial of anal dilatation, (iii) further trial of anal dilatation (with or without rectal biopsy), (iv) consideration for extended upper partial internal sphincterotomy.

Medical mangement. Those cases who had not previously been on regimens similar to our own were prescribed methylcellulose (Cologel) taken with a high fluid intake and standardized senna (Senokot). Often an initial clear out with rectal washouts, Micralax or phosphate enemata, were required. This regimen was continued for at least 2 to 3 months.

Anal dilatation. This was a very vigorous dilatation under general anaesthesia, up to 4 fingers of the operator's hand being introduced.

Upper partial sphincterotomy. After a trial of one or more anal dilatations, upper partial sphincterotomy was usually carried out on cases of proven ultrashort-segment Hirschsprung's disease, or on those cases with indistinguishable traces, the extent being determined by the physiological changes. A vertical left posterolateral incision was made which extended up through the affected segment, as indicated by the anorectal physiological studies, usually 4-5 $\mathrm{cm}$ from the anal verge.

Results of treatment of anal achalasia. 11 patients improved after medical management but those not responding to conservative treatment were given the following treatment (see Fig. 3). 

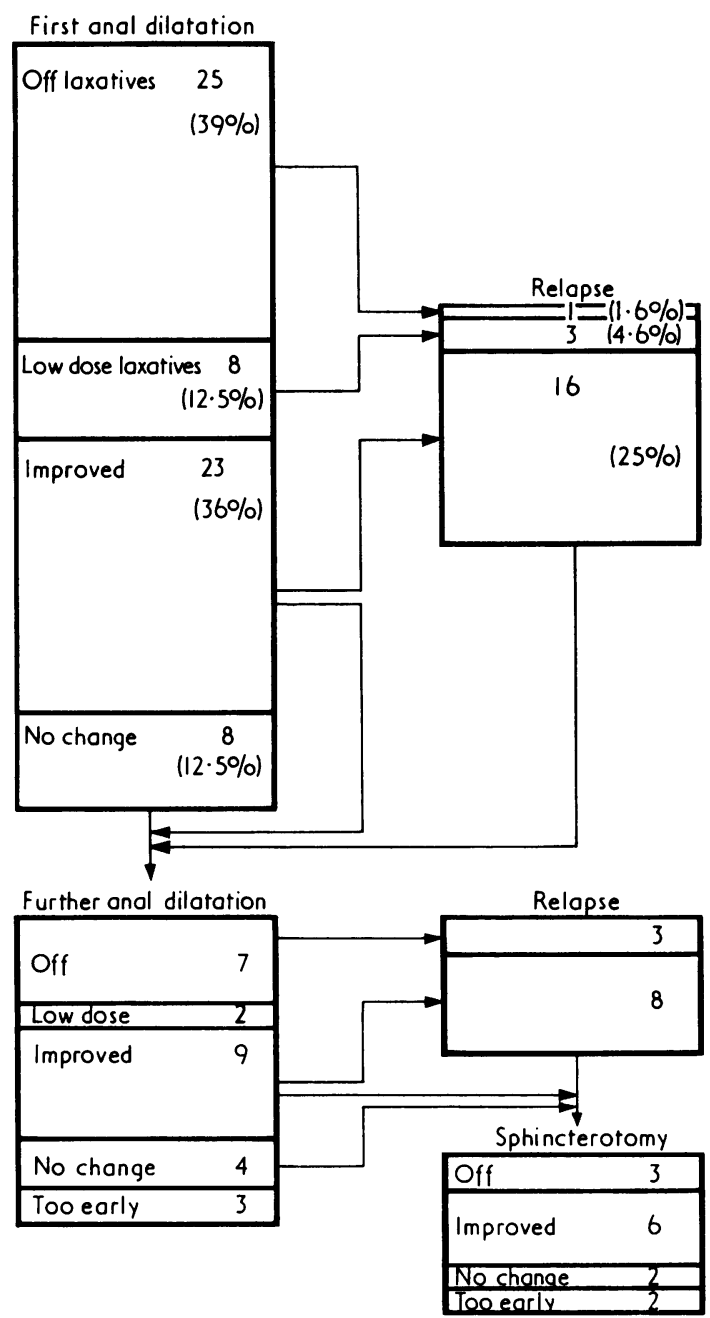

Fig. 3.-Results of anal dilatation.

Vigorous anal dilatation. $25(39 \%)$ responded dramatically to a single vigorous anal dilatation and were taken off all stimulant purgatives 1 to 2 months after this procedure. A regular bowel action could be obtained in a further $8(12.5 \%)$ with a much reduced dose of Senokot. $23(36 \%)$ showed increased ease in bowel action though still requiring reduced doses of purgatives but no enemata. In $8(12 \cdot 5 \%)$ no improvement was noted after anal dilatation. In a longer follow-up there were 16 relapses in the 23 cases showing only slight improvement, 3 in the group showing definite improvement, and only 1 in the group showing dramatic improvement.
Repeated anal dilatation. Those patients who relapsed or who showed no improvement after anal dilatation were subjected to one or more further anal dilatations, depending on clinical response. Dilatation was usually combined, under the same anaesthetic, with rectal biopsy. After repeat dilatation a further 7 children no longer required medication 3 months to 1 year after this procedure. Of these, 3 have subsequently relapsed. 2 further cases could be managed with reduced doses of standardized senna and methylcellulose. 9 severe cases improved to the extent that they no longer required repeated enemata; of these 8 later relapsed. 4 cases, in spite of initial response, were unchanged by subsequent dilatation.

The cases of ultrashort-segment Hirschsprung's disease responded in a similar manner to the cases in this group, responding initially to anal dilatation but soon relapsed.

Extended upper partial internal sphincterotomy. The cases with proven ultrashort-segment Hirschsprung's disease responded to an upper partial anal sphincterotomy, though some required more prolonged treatment for their megarectum. Of those not responding to, or relapsing, after repeated anal dilatation, 11 of the chronic constipation (nonHirschsprung) group were treated by sphincterotomy. 3 of these are off all purgatives, 6 were definitely improved, and 2 were unchanged.

Of those cases with chronic constipation not due to Hirschsprung's disease, 31 (48\%) were off all laxatives, $7(11 \%)$ were maintained on low doses, $13(20 \%)$ were improved, $3(5 \%)$ were unchanged, $10(16 \%)$ were still in course of treatment.

The behaviour problems in the majority of the children with demonstrable psychiatric factors settled with the treatment of their chronic constipation, many dramatically. For those whose psychiatric problems were more persistent, the help of a child psychiatrist and social worker was sought.

\section{Discussion}

It is clear that, when assessing a child with longstanding constipation, an organic cause for the constipation must be excluded. In this series 4 children had minor degrees of anal stenosis, 2 had sacral anomalies with neurogenic rectum and a minor degree of anal stricture. It is of interest that in our larger series of cases 2 children presented in one year with untreated covered anus at 4 and 11 years of age (Lawson, 1975). In all 6 of these cases the anus took the index finger and, in an objecting child, probably accounted for the failure of the anomaly to be previously recognized. Func- 
tional obstruction is more easily missed. In this series there were 10 cases of proven Hirschsprung's disease and a further 10 with traces physiologically indistinguishable from Hirschsprung's disease.

The physiological findings in the usual-length Hirschsprung's disease are now well established. The findings reported in 1967 (Lawson and Nixon), 1970 , and 1974 (Lawson) have been confirmed by other authors. Rhythmical activity, seen in the internal sphincter zone of the anal canal at rest showed a marked increase in amplitude and enhancement of the slow underlying fluctuation of this amplitude. In the ultrashort-segment Hirschsprung's disease spontaneous contraction waves were seen in the rectum, usually showing marked increase in amplitude; but, unlike the normal trace, were not accompanied by a corresponding fall in the internal sphincter pressure.

In the normal child rectal distension with small volumes is followed by a stimulated rectal wave, the pressure then returning to the resting level after the initial volumes. As the distending volume is increased, at a certain level ('critical volume') the rectal trace shows a persistent rise in pressure with superimposed, repeated, simple waves, continuing until evacuation occurs. In the usuallength Hirschsprung's disease, where the balloon lies in the segment, there is a rapid fall in pressure after distension, there being no stimulated wave. Distension is also often associated with pain. In short and ultrashort-segment disease the balloon lies above the segment. Simple stimulated waves are observed, but usually of increased amplitude, the rectum showing a markedly increased capucity.

In the internal sphincter zone of the normal child a simple stimulated rectal wave is accompanied by a fall in pressure (the trace showing an inverted wave form). After the initial volumes the pressure returns to resting level or above. When the critical volume is reached, and the rectal pressure rise is sustained, there is a persistent fall in pressure in the anal canal, though rectal pressure waves may be seen extending into the upper anal canal. This stage is accompanied by an imperative desire to defecate.

An intermediate stage may be noted where only the peaks of rectal pressure waves extended into the upper anal canal and are accompanied by less imperative or urgent desire to defecate. After evacuation there is an abrupt peak rise in pressure in the anal canal, associated with voluntary sphincter activity. There is also a slower rise in the internal spincter zone, with superimposed rhythmical waves. This rise, initially, reaches a level higher than the resting level, and then slowly returns to this level.
In Hirschsprung's disease no inhibition of pressure occurs in the internal sphincter zone. There appear to be two types of response (Lawson, 1970). In the first, a milder form of the disease, there is no rise in the baseline pressure in the internal sphincter zone, but prominent rhythmical activity continues unabated. In the second, the rectal distension is accompanied by a baseline rise and enhanced rhythmical activity. This type of response occurs in approximately 1 in 5 of the 'usual length' segment cases, but is found in the majority of 'ultrashort' (4-5 $\mathrm{cm}$ ) segment cases.

In 79 children the physiological traces suggested a functional obstructive element. The rectal traces, apart from showing a degree of megarectum, showed hypertrophy rather than inert rectal waves. Prominent rhythmical waves in the internal spincter traces were very similar to those seen in Hirschsprung's disease. The inhibitory response to a stimulated rectal wave was present but diminished or absent in the first $\mathrm{cm}$. Traces from the 4 cases with minor degrees of anal stricture were very similar, a string stricture being found at subsequent anal dilatation, $1 \mathrm{~cm}$ from the anal verge.

These findings suggest that the fault, in those with no stricture, may be in the first $\mathrm{cm}$. Anatomically, physiologically, and probably developmentally there appear to be two parts of the internal anal sphincter (Lawson, 1970). The lower $\frac{1}{3}$ of the anal canal lies within the lower part of the spincter (Lawson, 1974). Failure of adequate relaxation of this part with smooth muscle hypertrophy above (as with the first $\mathrm{cm}$ stricture) may account for the hypertrophic trace. The clinical findings and response to treatment also suggest that an obstructive element is present in the majority of these children. After initial clear out of inspissated stool the content is usually soft or semisolid in spite of the difficulty in evacuation, a consistent finding in short-segment Hirschsprung's disease. The dramatic response in $25(39 \%)$ cases to a single anal dilatation, in spite of many years' conservative management, is significant. A further $6(9 \%)$ responded to repeated dilatation and sphincterotomy. The cases of ultrashort-segment Hirschsprung's disease were cured by extended upper partial sphincterotomy. Of 11 cases not shown to have Hirschsprung's disease but not responding to anal dilatation, 3 were cured by sphincterotomy and 6 definitely improved.

Constipation (or faecal retention) and soiling are associated in many of these cases, soiling occurring in many cases with ultrashort-segment Hirschsprung's disease. The mechanism appears to be 
one of hypertrophic rectal contraction forcing soft or semisolid stools through the closed anal canal. One of us (J.O.N.L.) has recorded pressure waves of over $300 \mathrm{~cm}$ water from hypertrophied rectum above. As the anal canal contains the sensitive stratified cuboidal cell zone (Duthie and Bennett, 1963) the content is not felt until it is already being forced through the anal canal. A similar mechanism probably explains the majority of the cases shown not to have Hirschsprung's disease, even where gross faecal retention and overflow is not present. These are not children who pass a normal stool in an abnormal situation or place!

Psychiatric problems are present in many of these children, especially those with soiling. The fact that these problems improve dramatically in most of the children once the constipation and soiling is over suggests that these problems are secondary in character. A few may use their organic symptoms to effect, even cases with Hirschsprung's disease. They may need continued psychiatric help. Where no functional or organic obstruction is shown, many of the children give a history of neonatal problems or anal fissure. It is likely that the megarectum, initiated at this time, may set up a vicious circle. As the megarectum diminishes rectal sensation, the rectoanal inhibitory reflex is delayed holding the large bolus of stool off the sensitive transitional (or stratified cuboidal cell) zone, thus markedly delaying the sensation of imperative defecation. Holding back becomes possible especially with the memory of a painful fissure.

\section{Conclusion}

We have found that a single anal dilatation under a general anaesthetic cures approximately $40 \%$ of children with long-standing chronic constipation and helps most of the others; and of those relapsing, some have ultrashort-segment Hirschsprung's disease. We suggest that anal dilatation is a useful procedure in treatment and diagnosis of chronic constipation when medical means fail or cannot be withdrawn. Those failing to respond should be further investigated and the diagnosis of ultrashortsegment Hirschsprung's disease considered.

This work was supported by the Research and Endowments Fund of St. Thomas's Hospital and the Percy J. Neate Fellowship of the Clothworkers Company. We are grateful to Dr. B. D. R. Wilson and Professor J. W. Scopes and all those who referred cases of chronic constipation; to Miss Angela Brinton for technical assistance; to Mrs. Gale Willner for secretarial aid; and also to Mr. P. Bourne and the Medical Electronics Department of St. Thomas's Hospital.

\section{REFERENCES}

Denny-Brown, D., and Robertson, E. G. (1935). An investigation of the nervous control of defaecation. Brain, 58, 256

Duthie, H. C., and Bennett, R. C. (1963). The relation of sensation in the anal canal to the functional anal sphincter: a possible factor in anal continence. Gut, 4, 179 .

Gaston, E. A. (1948). Physiology of fecal continence. Surgery, Gynecology and Obstetrics, 87, 280.

Lawson, J. O. N. (1970). Structure and function of internal anal sphincter. Proceedings of the Royal Society of Medicine, 63, Suppl., 84.

Lawson, J. O. N. (1974). Pelvic anatomy. II. Anal canal and associated sphincters. Annals of the Royal College of Surgeons of England, 54, 288.

Lawson, J. O. N. (1975). The soiling child. Practitioner, 214, 807.

Lawson, J. O. N., and Nixon, H. H. (1967). Anal canal pressures in diagnosis of Hirschsprung's disease. Fournal of Pediatric Surgery, 2, 544.

Schapiro, O. (1948). Applied anatomy of infants and children in proctology. Review of Gastroenterology, 15, 307.

Schnaufer, L., Talbert, J. L., Haller, J. A., Reid, N. C. R. W. Tobon, F., and Schuster, M. M. (1967). Differential sphincteric studies in the diagnosis of ano-rectal disorders of childhood. fournal of Pediatric Surgery, 2, 538.

Schuster, M. M., Hookman, P., Hendrix, T. R., and Mendeloff, A. I. (1965). Simultaneous manometric recording of internal and external and sphincter reflexes. Bulletin of the fohns Hopkins Hospital, 116, 79.

Correspondence to Dr. G. S. Clayden, Department of Paediatrics, St. Thomas's Hospital, London SE1 7EH. 